\title{
WAVELET ANALYSIS OF THE LISBON AND GIBRALTAR NORTH ATLANTIC OSCILLATION WINTER INDICES
}

\author{
S. BARBOSA,* M. E. SILVA and M. J. FERNANDES \\ Faculdade Ciencias, Matematica Aplicada, Porto, Portugal \\ Received 18 April 2005 \\ Revised 29 July 2005 \\ Accepted 6 August 2005
}

\begin{abstract}
The North Atlantic Oscillation (NAO) is one of the most important climatic patterns in the Northern Hemisphere. Indices based on the normalised pressure difference between Iceland and a southern station, such as Lisbon or Gibraltar, have been defined in order to describe NAO temporal evolution. Although exhibiting interannual and decadal variability, the signals are statistically rather featureless and therefore it is difficult to discriminate between different types of stochastic models.

In this study, Lisbon and Gibraltar NAO winter indices are analysed using the discrete wavelet transform discrete wavelet transform(DWT). A multi-resolution analysis (MRA) is carried out for a scale-based description of the indices and the wavelet spectrum is used to identify and estimate long-range dependence. The degree of association of the two NAO indices is assessed by estimating the wavelet covariance for the two signals.

The scale-based approach inherent to the discrete wavelet methodology allows a scale-by-scale comparison of the signals and shows that although the short-term temporal pattern is very similar for both indices, the long-term temporal structure is distinct. Furthermore, the degree of persistence or 'memory' is also distinct: the Lisbon index is best described by a long-range dependent (LRD) process, while the Gibraltar index is adequately described by a short-range process. Therefore, while trend features in the Lisbon NAO index may be explainable by long-range dependence alone, with no need to invoke external factors, for the Gibraltar index such features cannot be interpreted as resulting only from internal variability through long-range dependence. Copyright (C) 2006 Royal Meteorological Society.
\end{abstract}

KEY WORDS: North Atlantic Oscillation; NAO; long-range dependence; stationary wavelet transform

\section{INTRODUCTION}

Climate low frequency variability tends to occur in large spatial patterns associated with changes in atmospheric circulation patterns (Trenberth, 1995). One of the most important modes of climate variability in the Northern Hemisphere is the North Atlantic Oscillation (NAO), characterised by a large-scale net displacement of atmospheric mass between the Arctic region and the middle latitudes (Wallace and Gutzler, 1981; Barnston and Livezey, 1987).

The NAO is associated with changes in the strength and path of storm systems crossing the Atlantic, changes in sea-ice cover, sea-surface temperature, wave heights and to temperature and precipitation anomalies over Europe, mainly in the winter season (Hurrell, 1995; Hurrell and van Loon, 1997; Rogers, 1997; Marshall et al., 2001; Wanner et al., 2001, Hurrell et al., 2003).

The spatial pattern of the NAO is described by a north-south dipole of pressure anomalies with one centre located over Greenland and another centre of opposite sign located in the Atlantic near Azores.

Indices based on the pressure difference between a station located in the southern centre of the dipole (Azores, Lisbon or Gibraltar) and a station in the northern centre (Stykkisholmur-Iceland) have been defined to characterise NAO. However, since the NAO is known to exhibit seasonal variability (Mächel et al., 1998; Trausti and Miles, 2001) it may not be adequately represented by an index based on two points at fixed

*Correspondence to: Susana Barbosa, Faculdade Ciencias, Matematica Aplicada, Porto, Portugal; e-mail: susana.barbosa@fc.up.pt 
locations. An alternative spatio-temporal description of the phenomenon is obtained from the leading EOF mode of monthly pressure fields north of $20^{\circ} \mathrm{N}$ weighted by area that yields a zonally more symmetric pattern, with one centre of action over the Arctic and an annular structure of opposite sign at mid-latitudes, referred to as the Arctic Oscillation (AO) or the Northern Hemisphere Annular mode (NAM) (Wallace, 2000; Thompson and Wallace, 2001). These two representations of the NAO phenomenon are associated with distinct dynamical concepts: the ocean/atmosphere interaction has a dominant role within the NAO paradigm, while the dynamics of the upper atmosphere associated with the polar vortex have a dominant role within the AO/NAM paradigm (Wallace, 2000; Wanner et al., 2001).

Spatio-temporal representations of the NAO are based on gridded pressure data, which are available over the entire North Atlantic for only a short time period. Station-based indices are based on data available back to the mid-nineteenth century and thus are more suitable for the investigation of NAO long-term temporal variability. A commonly used NAO index is the winter NAO index based on the differences between standardised December-March mean sea-level pressures at Lisbon and Stykkisholmur (Hurrell, 1995). Comparing pressure records from Iceland and the three southern stations, Pozo-Vasquez et al., (2000) concluded that Gibraltar is a better representation of the southern centre of the oscillation since a stronger out-of-phase relation is found for these stations. Recently, a new version for the Gibraltar index has been put forward by replacing Gibraltar data for the 1821-1856 period by observations from Cadiz/San Fernando stations (Vinther et al., 2003).

The NAO indices exhibit considerable inter-decadal variability, with long alternate periods of positive and negative phase dominance and a flat, slightly red, spectrum. Analysis of the winter NAO index has rejected a random walk for the description of the index temporal evolution (Wunch, 1999; Stephenson et al., 2000; Mills, 2004). There is still some debate, however, as to the type of model for the index since, owing to its featureless structure, it may be regarded as a realisation of several different stochastic processes. Stephenson et al., (2000) concluded that a long-range dependent (LRD) fractionally integrated noise provides a better fit to the NAO winter index than a stationary red noise, while Mills (2004) finds no evidence in favour of fractional integration for the time series. Characterisation of the NAO temporal structure is important for understanding the physical processes responsible for the observed variability, in particular, if the NAO trend results from an aggregation process, such as integration by the North Atlantic ocean of a large number of stochastic heat fluxes (Wunch, 1999; Stephenson et al., 2000), or if external forcing (Osborn, 2004) and other deterministic influences, such as ocean dynamics, influence NAO evolution (Greatbatch, 2000; Paeth et al., 2003; Hurrell et al., 2004).

The wavelet transform yields a flexible approach for the description of the local behaviour of a time series (Mallat, 1999; Percival and Walden, 2000). In this study, Lisbon and Gibraltar NAO winter indices are analysed using the discrete wavelet transform (DWT). The continuous wavelet transform (CWT) has been used in previous studies to obtain a scale-time decomposition of NAO and NAO-related signals (Higuchi et al., 1999; Keylock, 2003). However, the DWT is a more natural tool for a discrete signal such as the NAO index time series. The DWT eliminates the redundancy inherent to the CWT and it is easier to implement and is particularly useful to handle long-range dependence (Percival and Walden, 2000; Abry et al., 2003). Here the wavelet spectrum from a discrete wavelet-based analysis of variance is used to detect and estimate long-range dependence in the NAO indices.

\section{DATA}

Winter NAO indices based on standardised sea-level pressure differences from December to March between Stykkisholmur/Reykjavik and Lisbon (Hurrell, 1995) and between Stykkisholmur/Reykjavik and Gibraltar (Jones et al., 1997) are analysed. Although a longer index (back to 1821) is available using Gibraltar as the southern station, for comparison purposes only the 140-year period common to both indices, from 1864 to 2003 , is considered in the analysis.

Long-term variability, as described by a lowess (locally weighted regression) curve (Cleveland and Devlin, 1988) fitted to the data, is very similar for the two indices for the selected period, although variance is significantly lower for the Gibraltar index (Figure 1). 

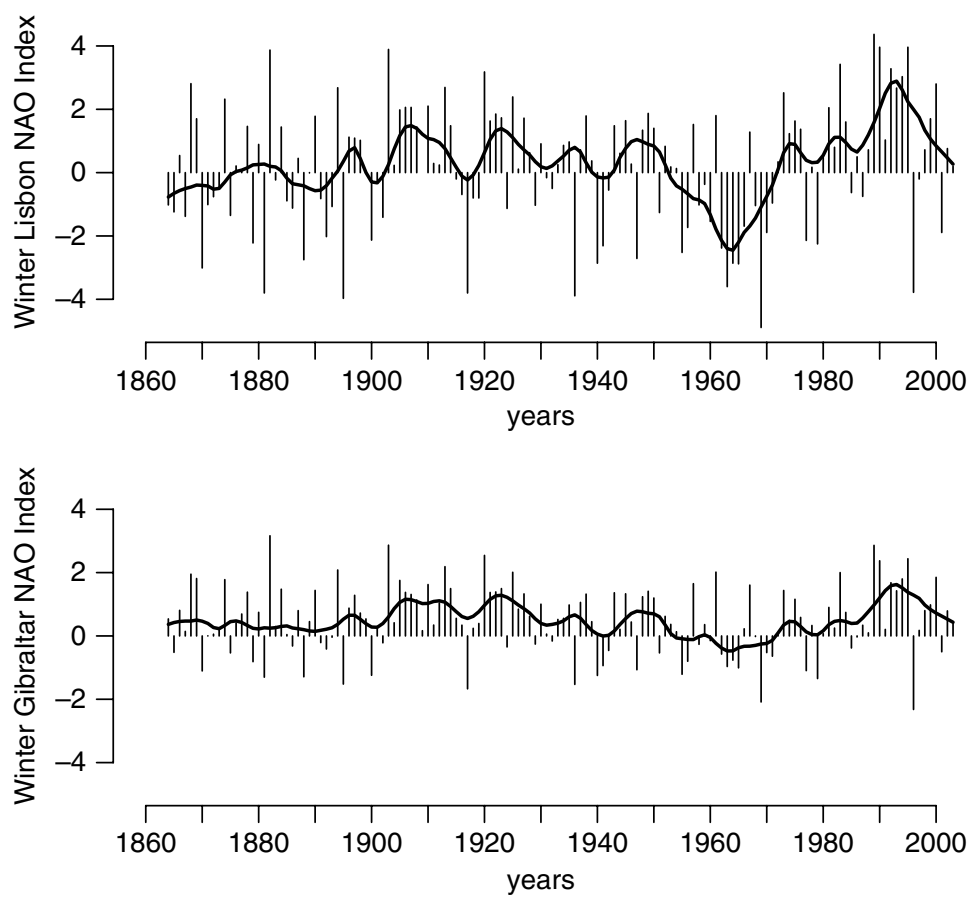

Figure 1. NAO indices (bars) and the lowess curve (solid line)

\section{ANALYSIS}

\subsection{Spectral analysis}

Spectral analysis is carried out for the two NAO indices. The cumulative periodogram and autocorrelation function are shown for each index in Figures 2 and 3 respectively. Autocorrelation values and spectral signals are within the confidence bands for the two time series, suggesting a white noise stationary process. A feature common to the two indices is the enhanced spectral power near 8 years (although not large enough to be statistically significant). There are, however, differences in the correlation structure of the two indices with the Lisbon index exhibiting a higher spectral content for very low frequencies. A jump in cumulative power is observed at frequency 0.13 (near 8 years) for the two indices and near 0 for the Lisbon index only.
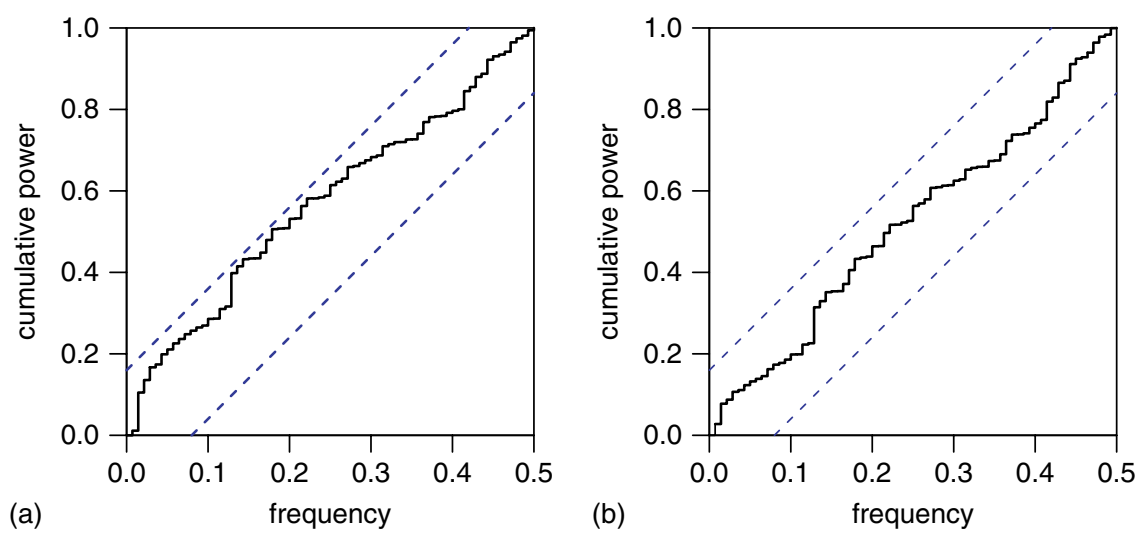

Figure 2. NAO index cumulative periodograms and corresponding 95\% significance bands. (a) Lisbon index and (b) Gibraltar index 

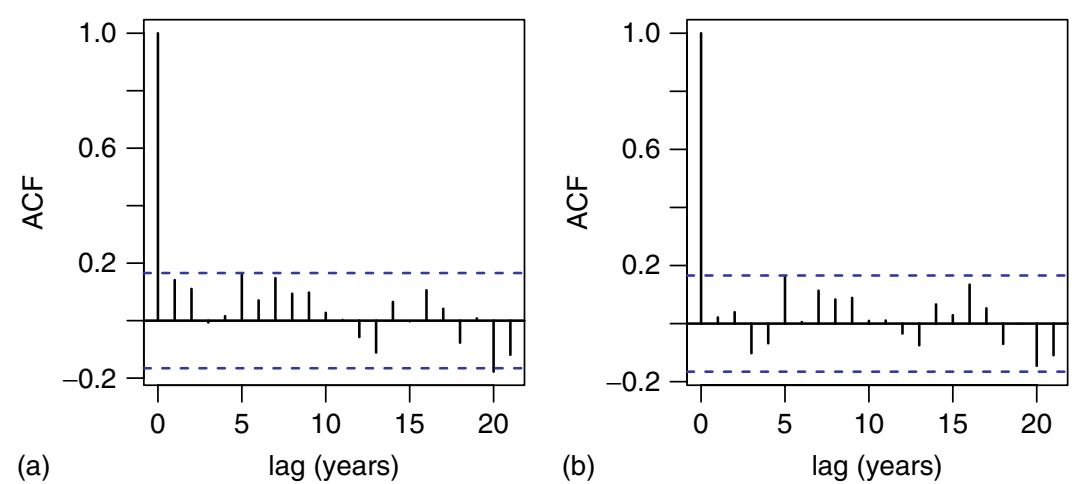

Figure 3. NAO index autocorrelation function (ACF) and corresponding 95\% significance bands. (a) Lisbon index and (b) Gibraltar index

Spectral estimates are also obtained using the non-parametric multi-taper method (MTM). MTM permits a reduction in the variance of spectral estimates by using a small set of tapers rather than the single spectral window (Ghil et al., 2002). The results obtained for each index from MTM analysis (Figure 4) are consistent with the results from periodogram spectral analysis. However, the MTM approach is able to identify significant signals in both series. For the Lisbon index, the largest spectral peak corresponds to a low frequency component (near frequency 0) and is significant at $99 \%$ significance level, while the next peak, corresponding to a period near 8.3 years, is only significant at $95 \%$ level. This situation is reversed for the Gibraltar index, since the dominant signal is not the low frequency component but the near 8.3-year cycle (significant at $99 \%$ level), and the low frequency peak is only significant at $95 \%$ significance level. Thus, although the same signals are present in both indices, the energy distribution is distinct for each index.

\subsection{Multi-resolution analysis}

The J-level DWT for a time series $X=\left\{X_{t}, t=1, \ldots, N\right\}$ is defined as an orthonormal transform $W=\tilde{W} \boldsymbol{X}$. The transformation matrix $W$ tilde can be grouped into $J+1$ submatrices $\tilde{W}=$ $\left[\tilde{W}_{1} \tilde{W}_{2} \ldots \tilde{W}_{j} \ldots \tilde{W}_{J} \tilde{V}_{J}\right]$, yielding a partition of the vector of coefficients $\boldsymbol{W}-\boldsymbol{W}$ into $J$ vectors of wavelet coefficients $\boldsymbol{W}_{j}$ and a vector of scaling (or approximation) coefficients $\boldsymbol{V}_{J}$ (Percival and Walden, 2000).

Partition of $\boldsymbol{W}$ defines a scale-by-scale additive decomposition (multi-resolution analysis, MRA)

$$
\boldsymbol{X}=\sum_{j=1}^{J} \tilde{W}_{j}^{T} \boldsymbol{W}_{\boldsymbol{j}}+\tilde{V}_{j}^{T} \boldsymbol{V}_{J}=\sum_{j=1}^{J} \boldsymbol{D}_{j}+\boldsymbol{S}_{J}
$$

where each detail $\boldsymbol{D}_{j}$ is associated with changes at physical scales of $\tau_{j}=2^{j-1}(j=1 \ldots, J)$ years, and the smooth $S_{J}$ (or approximation) represents variations over physical scales of $2^{J}$ years and higher. The details $D_{j}$ represent how (weighted) averages of the observations change from one time interval to the next and the scale $\tau_{j}$ gives the width of the time interval for which the averages are computed. Thus, detail $D_{j}$ represents differences in averages over time intervals of $2^{j-1}$ years corresponding to observations spaced between $2^{j}$ years.

Here, we use a modified version of the DWT, the maximal overlap discrete wavelet transform (MODWT), also called 'stationary DWT' (Nason and Silverman, 1995), 'undecimated DWT' (Shensa, 1992) or 'nondecimated DWT' (Bruce and Gao, 1996). The MODWT overcomes the sensitivity of the DWT to circular shifts of the series and can be applied to a time series of any length (unlike the DWT, which is only applicable to series of dyadic length) and yields more efficient estimates of wavelet variance and wavelet covariance (Percival and Walden, 2000).

A MRA based on a level $J=4$ MODWT is carried out for each NAO index time series (Figure 5) using a Daubechies Least Asymmetric filter of length 8, LA(8) (Daubechies, 1988), also called symlet8 (Bruce and 

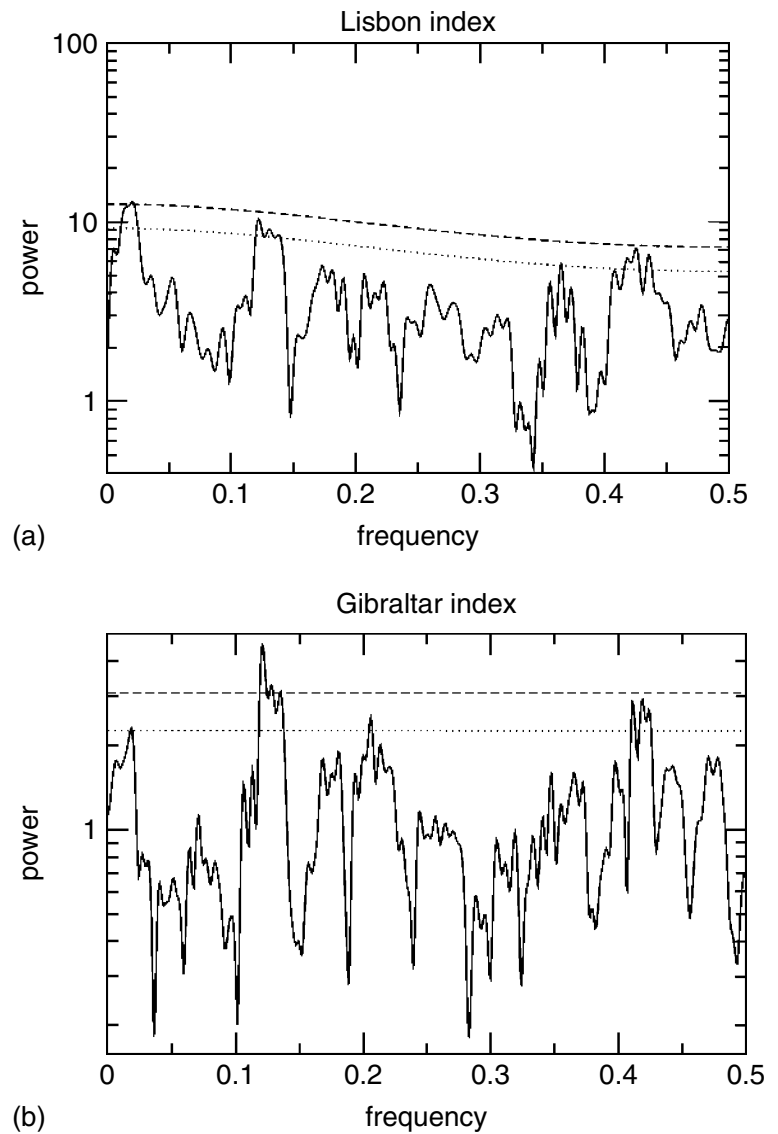

Figure 4. Multi-taper spectrum and 95\% (dotted) and 99\% (dashed) significance bands

Gao, 1996). Similar plots (not shown) are obtained when considering a wavelet with a higher number of vanishing moments such as LA(16) or symlet 16 .

Detail $\boldsymbol{D}_{1}$ reflects changes in the NAO index from one year to the next; bursts of increased variability occur near 1880, 1894 and 1995, corresponding to abrupt changes from high negative values in one year to high positive values in the next year (or vice versa); these are seen in retrospect in the time series plots (Figure 1), but are enhanced by the MRA. Detail $\boldsymbol{D}_{2}$ is associated with changes in physical scales of two years, with highest coefficients occurring around 1936 and 1970. Detail $\boldsymbol{D}_{3}$ corresponds to an oscillation with a period close to 8 years. This signal exhibits increased variability during the last 20 years of the series. Detail $\boldsymbol{D}_{4}$ reflects changes in physical scales of 8 years and is a low frequency oscillation with a period near 20 years. NAO variability over scales larger than 16 years is reflected by the smooth $\boldsymbol{S}_{4}$. The large-scale behaviour described by the smooth component is different for the two indices, particularly during the 1900-1940 period.

\subsection{Wavelet variance}

The DWT yields the following scale-based partition of energy:

$$
\|\boldsymbol{X}\|^{2}=\sum_{j=1}^{J}\left\|\boldsymbol{W}_{j}\right\|^{2}+\left\|\boldsymbol{V}_{J}\right\|^{2}
$$



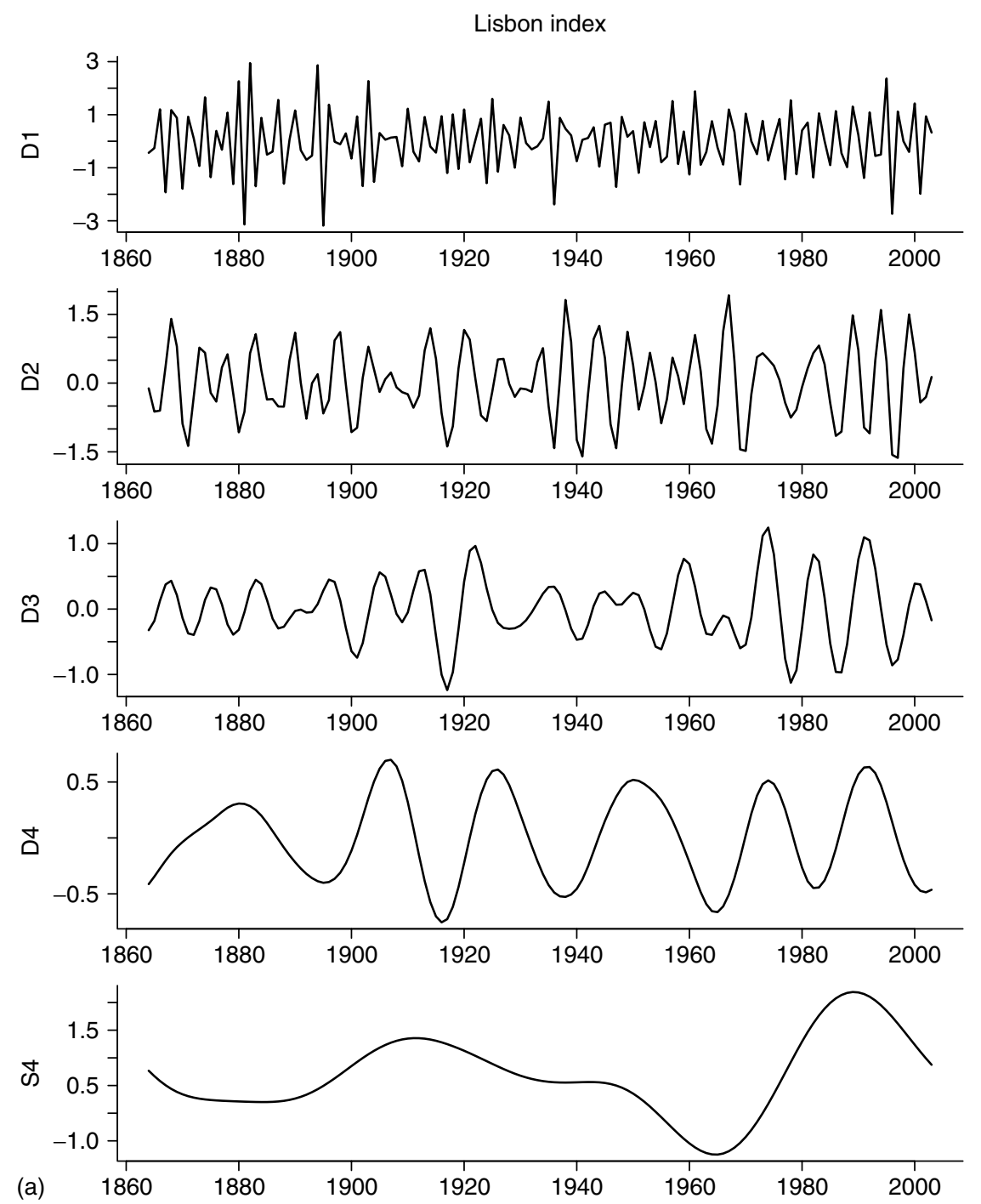

Figure 5a. MODWT-based multi-resolution analysis: wavelet details $\left(D_{j}\right)$ for levels $j=1$ to 4 representing changes on physical scales of $2^{(j-1)}$ years and smooth component (S4) reflecting variability over physical scales larger than $2^{4}$ years

Equivalent to the spectral density function that decomposes variance across frequencies, the wavelet variance decomposes variance across scales in a scale-based analysis of variance:

$$
\sigma_{X}^{2}=\sum_{j=1}^{J} v^{2}\left(\tau_{j}\right)
$$

where $v^{2}\left(\tau_{j}\right)$ is the wavelet variance at scale $\tau_{j}$. An unbiased estimator for the wavelet variance is given by (Percival and Walden, 2000):

$$
v^{2}\left(\tau_{j}\right)=\frac{1}{M_{j}} \sum_{t=1}^{N} \boldsymbol{W}_{j, t}^{2}
$$



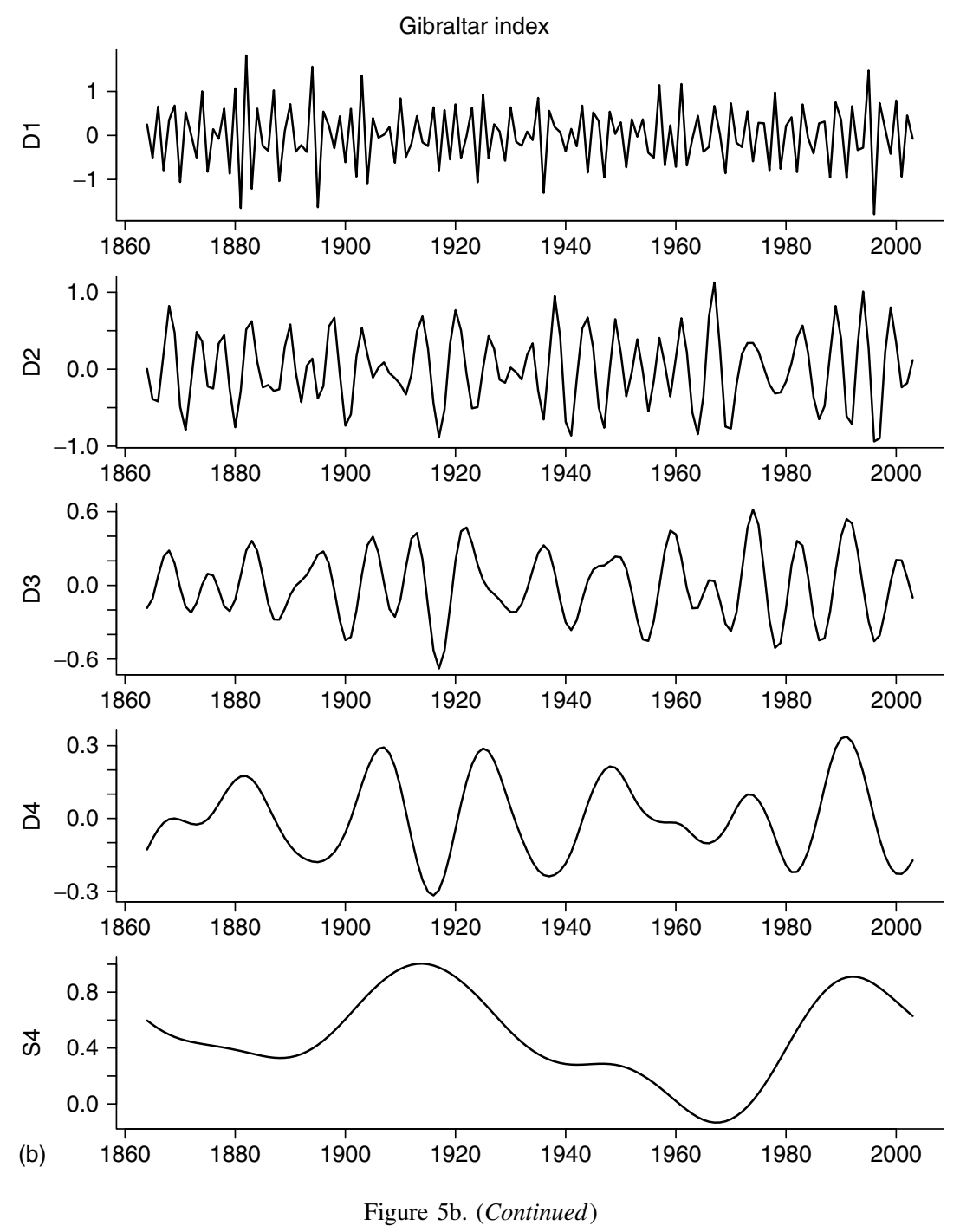

where $W_{j, t}$ denotes non-boundary wavelet coefficients and $M_{j}=N-\left(2^{j}-1\right)(L-1)$ is the number of non-boundary coefficients for a time series of length $N$ and a filter of width $L$.

Wavelet variance is estimated from the level $J=4$ MODWT transform for the winter NAO indices. Different wavelet filters and widths are considered to assess eventual non-stationarities and boundary effects: Haar filter $(L=2)$, Daubechies filters (also called daublets) of widths $L=4$ and $L=6(\mathrm{D}(4), \mathrm{D}(6))$ and a least asymmetric filter of width $L=8(\mathrm{LA}(8))$. For each filter, the estimated wavelet variance versus scale is plotted on a $\log 10-\log 10$ plot (Figure 6). The wavelet variance estimates are very similar for the four filters, indicating that the results are robust and are not influenced by boundary effects or non-stationarities. For further analysis, the Haar filter is selected since the smaller filter width guarantees a smaller number of coefficients affected by boundary conditions.

\subsection{Wavelet spectrum}

The wavelet variance of a process $X=\left\{X_{t}, t=1, \ldots, N\right\}$ over all dyadic scales $\tau_{j}=2^{j-1}(j=1, \ldots, J)$ constitutes a second order description of the process through a 'wavelet spectrum', with large values of $j$ corresponding to low frequencies and small values of $j$ corresponding to high frequencies. 

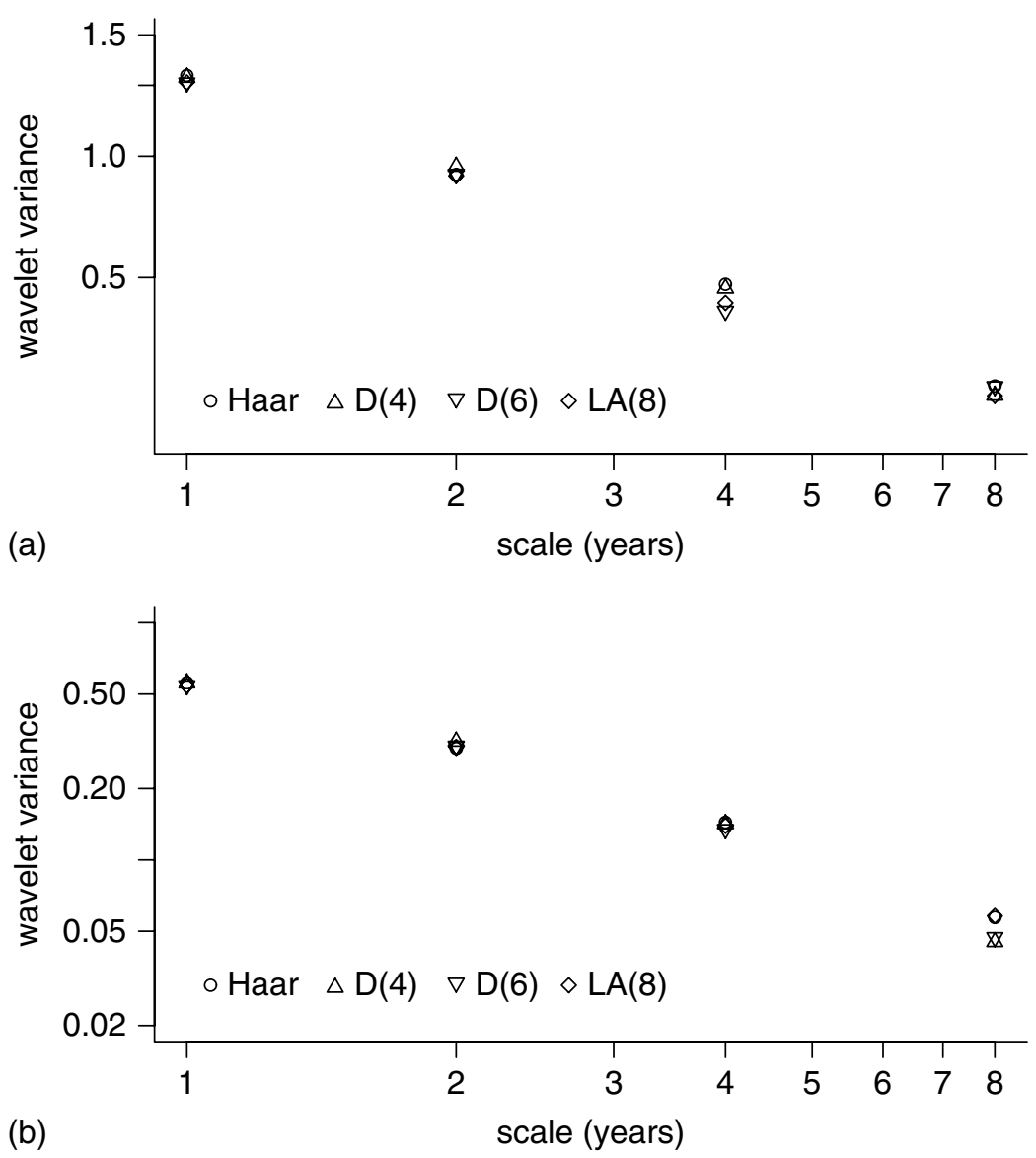

Figure 6. Log-log plot of wavelet variance versus scale for different wavelet filters (a) Lisbon index (b) Gibraltar index

The wavelet spectrum is estimated for each index from a level $J=5$ MODWT on the basis of the Haar wavelet filter. A higher level has been selected to guarantee a larger number of points in the wavelet spectrum, while complying with the restrictions to larger $J$ values from the time series size and the filter width.

The wavelet spectra exhibit a linear pattern (consistent with the 95\% confidence intervals) that is characteristic of a scaling process (Figure 7). The relation between wavelet variance and scale for a scaling process is approximately described by a power law and, therefore, in logarithmic coordinates by a straight line with slope $\beta$,

$$
\log \left(v^{2}\left(\tau_{j}\right)\right) \sim \zeta+\beta \log \left(\tau_{j}\right), j=l, l+1, \ldots, J l \geq 2
$$

The slope $\beta$ is estimated from the wavelet spectrum by weighted least squares (WLS, Table I) (Percival and Walden, 2000, p. 374).

\subsection{Long-range dependence}

A scaling process $X=\left\{X_{t}, t=1, \ldots, N\right\}$, is a long range dependent (LRD) or long memory (LM) process if its spectral density function $S_{X}$ is a power law for frequencies approaching zero:

$$
\lim _{f \rightarrow 0} S_{X}(f)=C|f|^{\alpha}
$$

where $C$ and $\alpha$ are constants satisfying $C>0$ and $-1<\alpha<0$. 

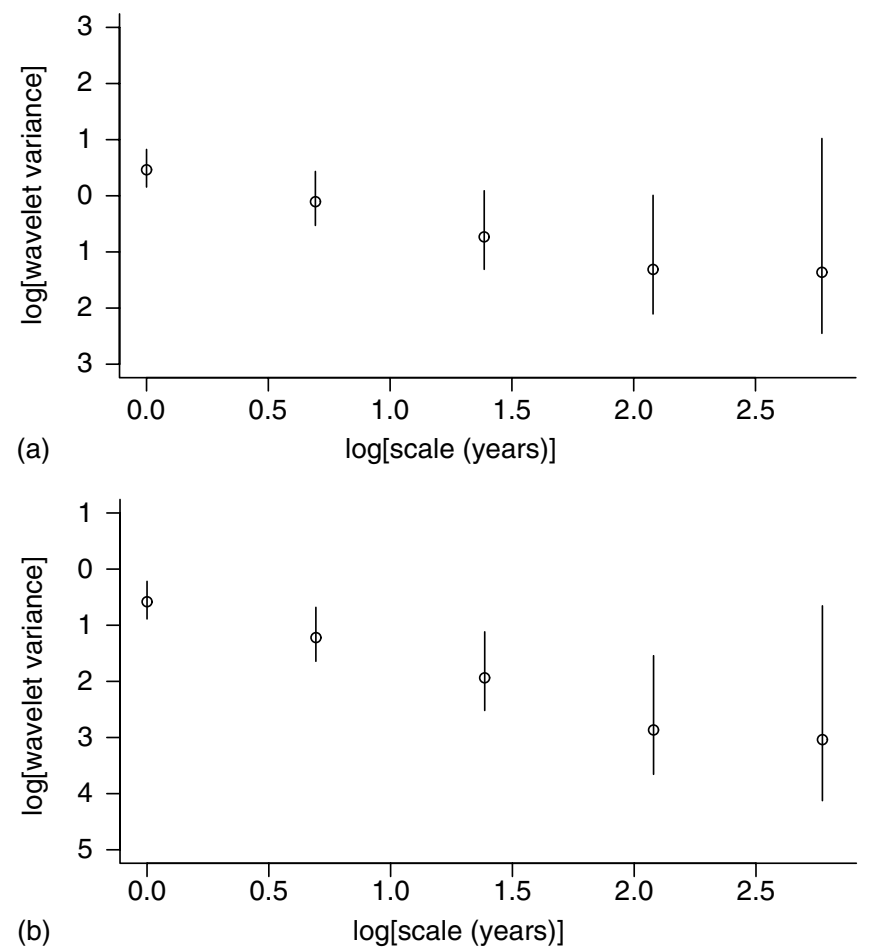

Figure 7. Wavelet spectrum and $95 \%$ confidence intervals. (a) Lisbon index and (b) Gibraltar index

Table I. Wavelet spectrum slope estimates for the Lisbon (LI) and Gibraltar (GI) indices

\begin{tabular}{lcc}
\hline Index & Slope estimate $(\beta)$ & Standard error \\
\hline LI & -0.71 & 0.21 \\
GI & -0.98 & 0.21 \\
\hline
\end{tabular}

The wavelet spectrum provides a summary of the spectral density function $S_{X}$, reproducing in the wavelet domain the power laws underlying the scaling processes (Abry et al., 2003). The slope $\beta$ of the wavelet spectrum is related to the scaling exponent $\alpha$ by

$$
\alpha=-\beta-1
$$

A time series model suitable for a process exhibiting long-range dependence is a fractionally differenced (FD) process

$$
(1-B)^{d} X_{t}=\varepsilon_{t} \varepsilon_{t} \sim N\left(0, \sigma_{\varepsilon}^{2}\right)
$$

The memory parameter $d$ of an FD process is related to the scaling exponent $\alpha$ by $d=-\alpha / 2$ and therefore to the wavelet spectrum slope by

$$
d=\frac{\beta+1}{2}
$$

Values of the LM parameter $d$ are computed for the two NAO indices from the slope estimates (Table II). The LM parameter for the Lisbon index is small but significantly different from zero, suggesting that a LRD 
Table II. Long memory parameter estimates for the Lisbon (LI) and Gibraltar (GI) indices

\begin{tabular}{lcc}
\hline Index & $d$ estimate & Standard error \\
\hline LI & 0.14 & 0.053 \\
GI & 0.01 & 0.053 \\
\hline
\end{tabular}

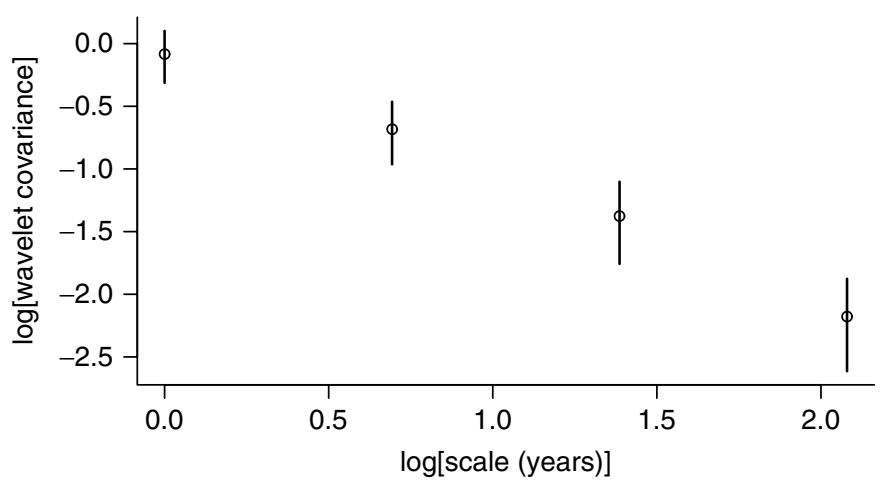

Figure 8 . Wavelet covariance and $95 \%$ confidence intervals

process is adequate for this time series, although dependence is weak. For Gibraltar, the value obtained for the LM parameter is not significantly different from zero, indicating that the index is adequately described by a short-range stationary process.

\subsection{Wavelet covariance}

The wavelet covariance gives a measure of the level of association between two time series on a scale-byscale basis (Lindsay et al., 1996; Whitcher et al., 2000). The wavelet covariance for two time series $X=\left\{X_{t}\right\}$, $\boldsymbol{Y}=\left\{Y_{t}\right\}$ at scale $\tau_{j}$ is defined as the covariance between the corresponding wavelet coefficients. An unbiased estimator for the wavelet covariance is given by

$$
\gamma_{X, Y}\left(\tau_{j}\right)=\frac{1}{M_{j}} \sum_{l=L_{j}-1}^{N-1} W_{j, l}^{X} W_{j, l}^{Y}
$$

where $W^{X}, W^{Y}$ are the non-boundary MODWT wavelet coefficients for processes $X$ and $Y$ at scale $\tau_{j}=2^{j-1}, M_{j}$ is the number of non-boundary coefficients and $L_{j}=\left(2^{j}-1\right)(L-1)+1$.

The wavelet covariance is estimated for the Lisbon and Gibraltar indices from non-boundary MODWT wavelet coefficients using the Haar filter (Figure 8). The wavelet covariance decreases with scale, indicating that the coherency between the two indices is higher at the smaller scales and lower at the larger scales. This is consistent with the inferences made from the MODWT-based multi-resolution analysis in terms of the Lisbon and Gibraltar indices being very similar for the first details but with distinct temporal patterns at larger scales.

\section{DISCUSSION AND CONCLUSIONS}

In this study, the low frequency properties of the Lisbon and Gibraltar NAO indices have been investigated. Although the time series seem to exhibit some interannual variability and decadal trends, the signals are 
statistically rather featureless, with autocorrelations and spectra typical of white noise processes. However, useful information may still be retrieved by using methodologies particularly adapted for handling short and noisy signals, such as multi-taper spectral estimation and wavelet analysis.

MTM spectra suggest a distinct low frequency behaviour for the two indices: the Lisbon index is dominated by a low frequency signal, significant at $99 \%$ significance level, while the Gibraltar index is dominated by a cycle with a period close to 8 years. This cycle is also identified in the Lisbon index (at 95\% significance level), and it is consistent with the spectral peak found by Wunch (1999) and the period for the cyclic component of the Basic Structural Model fitted by Mills (2004).

Details $D_{1}, D_{2}$ and $D_{3}$ from the MODWT-based multi-resolution analysis are very similar for the two indices, indicating a similar behaviour at small scales. Detail $D_{\mathbf{3}}$ exhibits increased variability in the final period of the time series, consistent with the intensification of the decadal signal proposed by Hurrell and van Loon (1997). The amplitude of each component decreases with scale, confirming inherent difficulties regarding the indices' predictability (Fernandez et al., 2003).

The smooth components for Lisbon and Gibraltar show significant differences in the low frequency properties of both indices, particularly during the 1900-1940 period. Comparison of the correlation between the pressure at the northern and southern stations indicates that this period corresponds to a weaker out-of-phase relation between Lisbon and Iceland owing to a dislocation southward of the Azores High (Mächel, 1998; Pozo-Vasquez et al., 2000). The smooth component obtained for the Lisbon index is consistent with the level component of a Basic Structural Model fitted to this series (Mills, 2004).

The wavelet spectrum exhibits a linear pattern, typical of a scaling process, i.e. a process for which a dominant characteristic scale is absent. A non-linear behaviour would be an indication of a multi-fractal scaling structure, but the investigation of multi-fractality would require higher (beyond second order) statistics (Riedi, 2003, for example).

The slope from the wavelet spectrum has been used to obtain an estimate for the scaling exponent $\alpha$ and, assuming an FD process, the LM parameter $d$. Since the estimated values of $\alpha$ are different from -2 , random walks are discarded as adequate processes for both time series, in agreement with Stephenson et al., (2000) and Mills (2004) for the Lisbon index. The LM parameter $d$ obtained for the Lisbon index $(d=0.14)$ is consistent with the maximum likelihood estimate of $d=0.13$ by Stephenson et al., (2000). For Gibraltar, the LM parameter is not significantly different from zero.

The wavelet covariance between the Lisbon and Gibraltar indices shows that the degree of association is not constant, with the coherency between the two signals decreasing with scale. Thus, on short timescales both indices are influenced by the same factors, giving a coherent description of the NAO phenomenon, while on the long-term timescales, the indices capture distinct aspects of the NAO, possibly owing to shifts in the position of the southern node of the NAO dipole at seasonal timescales and longer.

The analysis carried out here not only confirms findings from previous studies within a unified analysis framework based on the wavelet transform but also brings additional results concerning the temporal features of the analysed indices. The scale-based approach inherent to the discrete wavelet methodology allows a scale-by-scale comparison of the signals and shows that although the short-term temporal pattern is very similar for both indices, the long-term temporal structure is distinct. Furthermore, the degree of persistence or 'memory' is distinct for the Gibraltar and Lisbon indices: the Lisbon index is best described by a LRD process, while the Gibraltar index is adequately described by a stationary short-range process and does not exhibit long-range dependence.

The interest in the type of dependence present in the NAO indices is related to the detection and explanation of trends in the NAO. As pointed by Stephenson et al., (2000), care must be exercised when assessing and attributing causes to trend-like features in the NAO since these may be explainable by long-range dependence alone with no need to invoke external factors. We have shown that such LRD behaviour is not present in the Gibraltar index. Assuming that the Winter Gibraltar index is a more adequate station-based description of the NAO phenomenon (Pozo-Vasquez et al., 2000), our results indicate that trend features in the NAO cannot be interpreted as resulting only from internal factors through long-range dependence and, thus, external forcing cannot be ruled out. 
The physical interpretation of LRD behaviour is hampered by the multitude of possible generating mechanisms. One possible source is the aggregation of many (short-range) stationary processes. Such a mechanism has been proposed for the NAO through the aggregation of stochastic heat fluxes by the North Atlantic ocean (Wunch, 1999), but other possible sources of LRD behaviour include critical phenomena, associated with systems that are far from equilibrium (such as turbulent flows) or in equilibrium but very close to a critical point (Stanley et al., 2000, for example). Self-organised criticality has been proposed as a general framework for the generation of complex behaviour that would be expressed in the parameters of a physical system as long-range dependence (Bak, 1996), but this is still an open research question and an active area of debate.

From the perspective of practical applications, this analysis suggests that different outcomes may be expected from using the Lisbon or Gibraltar indices, particularly in analyses related to NAO low frequency temporal variability. The contrasting low frequency properties of the two indices also bear implications on NAO predictability and should be considered for optimal forecasting of NAO evolution.

This study has focused on two station-based representations of the NAO, the Lisbon and Gibraltar NAO indices, since these are used in many practical applications. A useful extension of this work would be the analysis, using the same methodology, of other representations for the NAO and other indices and NAO-related variables.

\section{ACKNOWLEDGEMENTS}

The Winter Lisbon index is from J. Hurrell (http://www.cgd.ucar.edu/ jhurrell/).

The Winter Gibraltar index is from P. Jones and T. Osborn (http://www.cru.uea.ac.uk).

Statistical analysis has been carried out with R software (http://R-project.org).

$\mathrm{R}$ package waveslim provided by B. Whitcher has been used for wavelet analysis.

MTM spectral analysis has been carried out with MTM-SSA toolkit provided by the MTM-SSA group (http://www.atmos.ucla.edu/tcd/ssa/).

This work has been supported by program POCTI through the Centro de Investigação em Ciências Geoespaciais (CICGE) of the Faculty of Science, University of Porto.

\section{REFERENCES}

Abry P, Flandrin P, Taqqu MS, Veitch D. 2003. Self-similarity and long range dependence through the wavelet lens. In Theory and Applications of Long Range Dependence, Doukhan P, Oppenheim G, Taqqu MS (eds). Birkhäuser: Boston; 527-556.

Bak P. 1996. How Nature Works: The Science of self-organised Criticality, Springer-Verlag: New York.

Barnston AG, Livezey RE. 1987. Classification, seasonality and persistence of low frequency atmospheric circulation patterns. Monthly Weather Review 115: 1083-1126.

Bruce AG, Gao H-Y. 1996. Applied Wavelet Analysis With S-PLUS. Springer-Verlag: New York.

Cleveland WS, Devlin SJ. 1988. Locally-Weighted fitting: An approach to fitting analysis by local fitting. Journal of the American Statistical Association 83: 596-610.

Daubechies I. 1988. Orthonormal bases of compactly supported wavelets. Communications on Pure and Applied Mathematics 41: 909-996.

Fernandez I, Hernandez C, Pacheco JM. 2003. Is the North Atlantic Oscillation just a pink noise. Physica A 323: 705-714.

Ghil M, Allen MR, Dettinger MD, Ide K, Kondrashov D, Mann ME, Robertson AW, Saunders A, Tian Y, Varadi F, Yiou P. 2002. Advanced spectral methods for climatic time series. Reviews of Geophysics 40: 1-41.

Greatbatch RJ. 2000. The North Atlantic Oscillation. Stochastic Environmental Research and Risk Assessment 14: $213-242$.

Higuchi K, Huang J, Shabbar A. 1999. A wavelet characterisation of the North Atlantic Oscillation variation and its relationship to the North Atlantic Sea surface temperature. International Journal of Climatology 19: 1119-1129.

Hurrell JW. 1995. Decadal trend in the NAO: regional temperatures and precipitation. Science 269: 676-679.

Hurrell JW, van Loon H. 1997. Decadal variations in climate associated with the NAO. Climatic Change 36: 301-326.

Hurrell JW, Kushnir Y, Ottersen G, Visbeck M. 2003. An overview of the North Atlantic Oscillation in North Atlantic Oscillation, Climate significance and environmental impact. Geophysical Monograph 134: 1-35.

Hurrell JW, Hoerling MP, Phillips AS, Xu T. 2004. Twentieth century North Atlantic climate change. Part I: Assessing determinism. Climate Dynamics 23: 371-389.

Jones PD, Jonsson T, Wheeler D. 1997. Extension to the North Atlantic Oscillation using early instrumental pressure observations from Gibraltar and South-West Iceland. International Journal of Climatology 17: 1433-1450.

Keylock CJ. 2003. The North Atlantic Oscillation and snow avalanching in Iceland. Geophysical Research Letters 30: 1254, DOI:10.1029/2002GL016272. 
Lindsay RW, Percival DB, Rothrock DA. 1996. The discrete wavelet transform and the scale analysis of the surface properties of sea ice. IEEE Transactions on Geoscience and Remote Sensing 34: 771-787.

Mächel H, Kapala A, Flohn G. 1998. Behaviour of the centres of action above the Atlantic since 1881. Part I: characteristics of seasonal and interannual variability. International Journal of Climatology 18: 1-22.

Mallat S. 1999. A Wavelet Tour of Signal Processing. Academic Press: San Diego, California.

Marshall J, Kushnir Y, Battisti D, Chang P, Czaja A, Dickson R, Hurrell JW, McCartney M, Saravanan R, Visbeck M. 2001. North Atlantic Climate Variability: Phenomena, Impacts and mechanisms. International Journal of Climatology 21: 1862-1898.

Mills TC. 2004. Is the North Atlantic Oscillation a random walk? A comment with further results. International Journal of Climatology 24: $277-283$.

Nason GP, Silverman B. 1995. The stationary wavelet transform and some statistical applications. In Wavelets and Statistics, Antoniadis A, Oppenheim G, (eds). Springer-Verlag: New York, 281-299.

Osborn TJ. 2004. Simulating the winter North Atlantic Oscillation: the roles of internal variability and greenhouse gas forcing. Climate Dynamics 22: 605-623.

Paeth H, Latif M, Hense A. 2003. Global SST influence on twentieth century NAO variability. Climate Dynamics 21: 63-75.

Percival D, Walden A. 2000. Wavelet Methods for Time Series Analysis. Cambridge University Press: Cambridge.

Pozo-Vasquez D, Esteban-Parra MJ, Rodrigo FS, Castro-Diez Y. 2000. An analysis of the variability of the North Atlantic Oscillation in the time and the frequency domains. International Journal of Climatology 20: 1675-1692.

Riedi RH. 2003. Multifractal Processes. In Theory and Applications of Long Range Dependence, Doukhan P, Oppenheim G, Taqqu MS (eds). Birkhäuser: Boston; 625-716.

Rogers J. 1997. North Atlantic storm-track variability and its association to the NAO and climate variability of Northern Europe. Journal of Climate 10: $1635-1647$.

Shensa MJ. 1992. The discrete wavelet transform: wedding the à Trous and Mallat algorithms. IEEE Transactions on Signal Processing 40: $2464-2482$.

Stanley HE, Amaral LN, Gopikrishnan P, Ivanov PC, Keitt TH, Plerou V. 2000. Scale invariance and universality: organising principles in complex system. Physica A 281: 60-68.

Stephenson D, Pava V, Bojariu R. 2000. Is the North Atlantic Oscillation a random walk? International Journal of Climatology 20 : $1-18$.

Thompson D, Wallace J. 2001. Regional climate impacts of the Northern Hemisphere Annular Mode and associated climate trends. Science 293: 85-89.

Trausti J, Miles MW. 2001. Anomalies in the seasonal cycle of sea level pressure in Iceland and the North Atlantic Oscillation. Geophysical Research Letters 28: 4231-4234.

Trenberth KE. 1995. Atmospheric circulation climate changes. Climatic Change 31: 427-453.

Vinther BM, Andersen KK, Hansen AW, Schmith T, Jones PD. 2003. Improving the Gibraltar/Reykjavik NAO index. Geophysical Research Letters 30: 2222, DOI:10.1029/2003GL018220.

Wallace JM. 2000. North Atlantic Oscillation/Annular mode: Two paradigms - one phenomenon. Quarterly Journal of the Royal Meteorological Society 126: 791-805.

Wallace JM, Gutzler SS. 1981. Teleconnections in the geopotential height field during the Northern Hemisphere winter. Monthly Weather Review 109: 784-812.

Wanner H, Bronnimann S, Casty C, Gyalistras D, Luterbacher J, Schmutz C, Stephenson DB, Xoplaki E. 2001. North Atlantic Oscillation- concepts and studies. Surveys in Geophysics 22: 321-382.

Whitcher B, Guttorp P, Percival DB. 2000. Wavelet analysis of covariance with application to atmospheric time series. Journal of Geophysical Research 105: 14941-14962.

Wunch C. 1999. The interpretation of short climatic records. Bulletin of the American Meteorological Society 80: 245-255. 